\title{
Intraruminal soluble glass boluses containing melatonin can induce early onset of ovarian activity in ewes
}

\author{
A. L. Poulton, A. M. Symons, M. I. Kelly* and J. Arendt \\ Department of Biochemistry, University of Surrey, Guildford, Surrey GU2 5XH, and \\ * Research and Development Laboratories, Pilkington Brothers p.l.c., Lathom, Ormskirk,
} Lancashire L40 5UF, U.K.

\begin{abstract}
Summary. Soluble phosphate glass boluses (preparations A, B, and C) have been developed to release melatonin into the reticulo-rumen of ewes at relatively different (fast, medium or slow) rates. A fourth type (preparation D) containing no melatonin was used as a sham control. Four groups of seasonally anoestrous Suffolk-cross ewes were respectively dosed with preparations A, B, C or D on 4 July. Plasma samples were collected twice weekly for melatonin and progesterone assay.

In Groups A, B and C, elevated daytime plasma concentrations of melatonin could be detected for about 5 weeks after bolus administration. However, the pattern of hormone release was variable between groups, with Group $\mathrm{C}$ animals maintaining higher plasma melatonin concentrations for a longer period. The control animals had undetectable daytime melatonin levels. The onset of cyclic ovarian activity in the animals treated with the 'slow' release bolus (Group $C$ ) was significantly $(P<0.05)$ advanced compared to the control group. The 'fast' and 'medium' release treatments (Groups A and B) did not significantly alter the onset of ovarian activity.

The results indicate the potential of a novel and convenient method of melatonin delivery for induction of early breeding activity in ewes.
\end{abstract}

\section{Introduction}

Secretion of the pineal hormone melatonin mediates the effect of changing photoperiod upon seasonal reproductive function in the ewe (see reviews by Bittman, 1984; Kennaway, 1984). Timed administration of supplementary melatonin by feeding (Kennaway et al., 1982; Arendt et al., 1983), injection (Nett \& Niswender, 1982) or infusion (Bittman \& Karsch, 1984) can stimulate an early onset of breeding activity by pharmacologically mimicking the onset of short photoperiodic conditions. Continuous melatonin administration via subcutaneous (English et al., 1986) or vaginal implants (Nowak \& Rodway, 1985) is probably perceived as a 'very short-day' response (Lincoln \& Ebling, 1985) and can also induce early breeding activity.

In breeds such as the Suffolk-cross and Mule, both timed and continuous melatonin treatments are only effective around mid-summer, probably following exposure to a critical interval of long photoperiod (Nowak \& Rodway, 1985; English et al., 1986). In the less seasonal Merino crossbreeds, melatonin treatment can be effective before the summer solstice (Kennaway et al., 1982) and can induce a relative increase in ovulation rate (Kennaway et al., 1984).

It is clear that any convenient and inexpensive method of melatonin administration not only provides a useful research tool but would be of practical benefit to the commercial sheep industry.

The ruminal stomach provides an ideal site for controlled delivery preparations (Telfer, 1984; Cardinal, 1985) which, by virtue of their size, shape and density are normally retained in the reticulum. This study describes performance data of a biodegradable soluble glass/melatonin device developed for oral administration in the ewe. Containing the same amount of melatonin, 3 types of 
bolus designed to release the hormone within the rumen at different rates, were administered to seasonally anoestrous Suffolk-cross ewes in mid-summer. Daytime plasma melatonin concentrations provided an index of the release pattern of melatonin from the ruminal boluses whilst cyclic ovarian activity was indicated by plasma progesterone profiles.

\section{Materials and Methods}

Melatonin/soluble glass boluses. Polymer-bonded soluble phosphate glass boluses incorporating powdered melatonin (150 mg) (Sigma Chemical Corporation, Poole, Dorset, U.K.) were prepared at the Research and Development Laboratories, Pilkington Brothers p.l.c. in collaboration with the Department of Biochemistry, University of Surrey. Each bolus was cylindrical in shape (diameter $1.8 \mathrm{~cm}$, length $3.0 \mathrm{~cm}$ ) with an average mass of $23 \mathrm{~g}$, resulting in an average specific gravity of 3.0. Variation in the polymer-bonded soluble glass formulation alters the aqueous solubility of the bolus which in turn determines the release-rate of melatonin. Preliminary in-vitro studies (data not published) indicated 3 formulations (preparations A, B and C) that would dissolve within the rumen at relatively different (fast, medium and slow) rates. A fourth type of bolus (preparation D) containing no melatonin was developed as a sham-control treatment.

Experimental animals. Sixteen maiden Suffolk-cross ewes, born in spring 1984, were maintained under natural photoperiod at the MAFF Central Veterinary Laboratories (CVL), Weybridge, Surrey (latitude $51^{\circ} 41^{\prime} \mathrm{N}$ ). The animals were penned together in a large open shed within an area measuring $10 \times 5$ metres and were fed a ration of sheep rearer nuts (Southern Counties Agricultural Trading Society, Godalming, Surrey, U.K.) at 230 g/ewe/day and allowed free access to hay and water.

The ewes were randomly divided into four groups of four and on 4 July 1985 (Day 0) were respectively dosed (oesophageal balling gun no. V687A, Arnolds Veterinary Products, Reading, Berkshire, U.K.) with preparations A, $\mathrm{B}, \mathrm{C}$ or $\mathrm{D}$. The animals were weighed 2 days before and 116 days after treatment.

Blood sampling. From 28 June to 31 October 1985, blood samples $(10 \mathrm{ml})$ were collected between 09:00 and 11:00 h twice weekly by jugular venepuncture using heparin-coated vacutainers (Becton Dickinson Ltd, Cowley, Oxfordshire, U.K.) and the plasma stored at $-20^{\circ} \mathrm{C}$ until hormone assay.

Melatonin assay. Plasma melatonin was estimated by a direct radioimmunoassay (RIA) (Fraser et al., 1983) as described previously (Poulton et al., 1986). Plasma samples of 25 , 100 and $478 \mathrm{pg}$ melatonin/ml had inter-assay coefficients of variation of 21,19 and $13 \%$ respectively and intra-assay coefficients of variation of 12,12 and $7 \%$ respectively. The limit of assay sensitivity, as expressed as the value of two standard deviations below maximum (zero melatonin standard) binding, was $4 \mathrm{pg} / \mathrm{ml}$ for $500 \mu \mathrm{l}$ plasma assayed.

Progesterone assay. Plasma progesterone was estimated by a direct enzyme-linked immunoabsorbent assay (ELISA) (Sauer et al., 1986) as described previously (English et al., 1986). Plasma samples of 0.2 and $1.5 \mathrm{ng}$ progesterone/ml had inter-assay coefficients of variation of 23 and $13 \%$ respectively and intra-assay coefficients of variation of 3 and $4 \%$ respectively. The limit of assay sensitivity, expressed as the value of two standard deviations below maximum (zero progesterone standard) binding, was $0.1 \mathrm{ng} / \mathrm{ml}$ for $20 \mu \mathrm{l}$ plasma assayed.

Reproductive activity. To distinguish between 'normal' and 'short luteal phase' oestrous cycles, the former were defined as a series of at least 3 plasma samples with detectable progesterone concentrations, providing at least one of these samples had a progesterone concentration $>1.0 \mathrm{ng} / \mathrm{ml}$. The onset of cyclic ovarian (breeding) activity was defined as the occurrence of at least 2 consecutive 'normal' cycles.

Statistical analysis. Values are given as mean \pm s.e.m. Daytime plasma melatonin concentrations in Groups A, B and $\mathrm{C}$, during the period 1-33 days after bolus administration, were analysed using two-way analysis of variance and tested at each blood sampling episode using one-way analysis of variance. Onset of ovarian activity between all groups was assessed by one-way analysis of variance. Differences between treatment groups were tested using Duncan's new multiple range test at 2 levels of significance $(P<0.05$ and $P<0.01)$.

\section{Results}

Pooled plasma melatonin and individual plasma progesterone concentrations are presented in Fig. 1.

In Groups A, B and C, daytime plasma melatonin concentrations $>10 \mathrm{pg} / \mathrm{ml}$ could be detected until 33-40 days after treatment. Group D ewes had undetectable levels throughout the study. During Days 1-33 (Day $0=$ day of treatment) there was no difference in overall melatonin concentrations between Groups $\mathrm{A}, \mathrm{B}$ and $\mathrm{C}$ although there was variation over time $(P<0.002)$. 


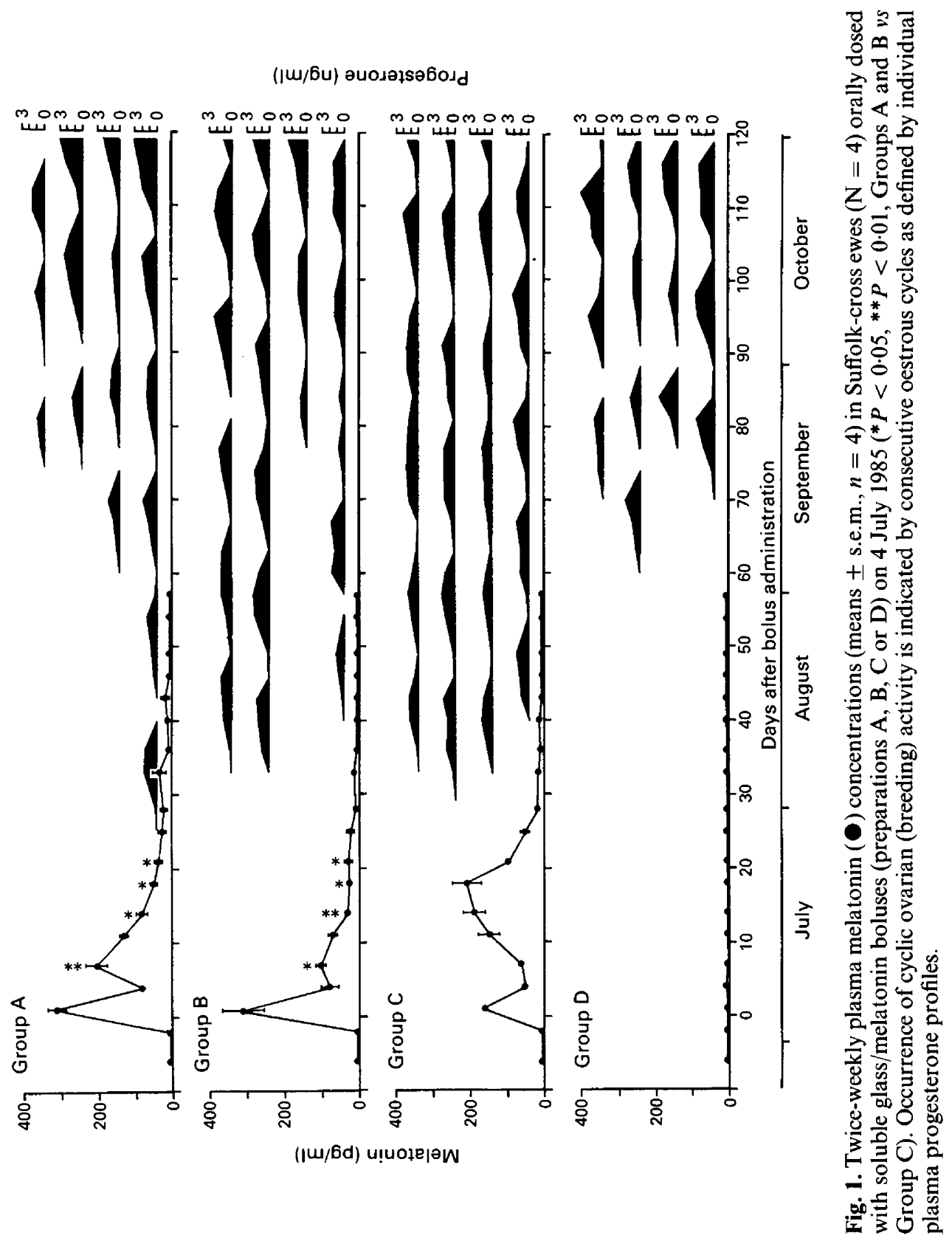


The plasma melatonin profile was similar within groups and biphasic in nature. In Groups A and B, melatonin peaks of about 300 and $150 \mathrm{pg} / \mathrm{ml}$ occurred at Days 1 and 7. In Group C, peaks of about 180 and $200 \mathrm{pg} / \mathrm{ml}$ occurred at Days 1 and 18. At Days 14, 18 and 21, plasma melatonin concentrations were significantly greater in Group C (Fig. 1).

There was a significant group effect in the onset of cyclic ovarian activity $(P<0.03)$ with the mean date of onset in Group C (9 August, $36 \pm 3$ days) significantly advanced compared to that of the control group (14 September, $72 \pm 3$ days) $(P<0.05)$. The start of ovarian cyclicity was not advanced in Groups A (3 September, $61 \pm 11$ days) or B (21 August, $48 \pm 10$ days). There were no other significant interactions.

All groups displayed a similar weight increase over the 4-month experimental period. Weights (overall mean \pm s.e.m. values) of the ewes, 2 days before and 116 days after treatment, were $55 \pm 2$ and $61 \pm 2 \mathrm{~kg}$, respectively.

\section{Discussion}

All animals in Groups A, B and C displayed elevated daytime plasma concentrations of melatonin for approximately 5 weeks, indicating that, while dissolving in the rumen, these preparations released (immunologically active) hormone which was taken up into the peripheral blood system over this period. As endogenous melatonin is mainly secreted nocturnally, it was assumed that daytime plasma levels could be attributed to the treatments.

It would appear that no boluses were regurgitated, indicating that the bolus mass was sufficient to be retained in the ruminal system and the consistent plasma melatonin profile within each treatment group suggests that the boluses were uniformly and not prematurely degraded. All animals gained weight over the experimental period suggesting an absence of any adverse treatment effects.

Plasma concentrations in Group C ewes were markedly elevated $(>50 \mathrm{pg} / \mathrm{ml}) 11-25$ days after bolus administration compared to those in the other groups. It would appear that this extended period of elevated plasma hormone concentration has contributed to the consistent early onset of ovarian activity in these animals. The critical (daytime) plasma melatonin concentration and the duration of such treatment required to mimic effectively a short photoperiodic response in ewes remain to be accurately defined. This study suggests that at least nocturnal concentrations (i.e. $30-200 \mathrm{pg} / \mathrm{ml}$ ) for approximately 5 weeks are needed, although supraphysiological levels over a longer period are also effective (Hsieh \& Chien, 1985).

The sham-control ewes exhibited the start of ovarian activity earlier than expected of Suffolkcross ewes in this locality (Arendt et al., 1983; English et al., 1986). Within an enclosed area, ewes displaying early cyclic activity may (via pheromonal cues?) stimulate a similar but premature response in other (untreated) animals. In an 'early breeding' flock, this is actually a desirable phenomenon but may confound experimental treatment versus control comparisons. This issue should be considered in further studies of this nature.

Caution should also be exercised in drawing conclusions from a study that was limited in group size and restricted to one breed. However, these preliminary results provide a positive indication of the efficacy of this technique. Field trials to establish the performance of the soluble glass/ melatonin bolus with respect to induction of early oestrus and subsequent lambing rates are currently underway. Similar trials have been extended to the goat and deer.

A biodegradable intraruminal device capable of sustaining elevated plasma melatonin concentrations would provide a novel and inexpensive method of inducing those seasonal responses in ruminants associated with short photoperiod.

We thank Mr Phil Irlam, Mr Ted White and Dr Brian Algar of Pilkington Brothers plc for their participation in the development of the melatonin/soluble-glass bolus; $\mathrm{Mr}$ Ben Parker and $\mathrm{Mr}$ Tony Quinn of MAFF, CVL, Weybridge, for their management of the experimental animals; and 
Dr John Foulkes of MAFF, Cattle Breeding Centre, Shinfield, for reagents and assistance with the progesterone assay. This work was sponsored by Pilkington Brothers plc, U.K. and is published with the permission of the Directors and Mr A. S. Robinson, Director of Group Research and Development.

\section{References}

Arendt, J., Symons, A.M., Laud, C.A. \& Pryde, S.J. (1983) Melatonin can induce the early onset of the breeding season in ewes. J. Endocr. 97, 395-400.

Bittman, E.L. (1984) Melatonin and photoperiodic time measurement: Evidence from rodents and ruminants. In The Pineal Gland, pp. 155-192. Ed. R. J. Reiter. Raven Press, New York.

Bittman, E.L. \& Karsch, F.J. (1984) Nightly duration of pineal melatonin secretion determines the reproductive response in inhibitory daylength in the ewe. Biol. Reprod. 30, 585-593.

Cardinal, J.R. (1985) Controlled drug delivery: veterinary applications. $J$. contr. Release 2, 393-403.

English, J., Poulton, A.L., Arendt, J. \& Symons, A.M. (1986) A comparison of the efficiency of melatonin treatments in advancing oestrus in ewes. J. Reprod. Fert. 77, 321-327.

Fraser, S., Cowen, P., Franklin, M., Franey, C. \& Arendt, J. (1983) Direct radioimmunoassay for melatonin in plasma. Clin. Chem. 29, 396-397.

Hsieh, D.S.T. \& Chien, Y.W. (1985) Enhanced release of drugs from silicone elastomers (III). Subcutaneous controlled administration of melatonin for early onset of estrus cycles in ewes. Drug Development and Industrial Pharmacy 11, 1433-1446.

Kennaway, D.J. (1984) Pineal function in ungulates. Pineal Research Reviews 2, 113-141.

Kennaway, D.J., Gilmore, T.A. \& Seamark, R.E. (1982) Effect of melatonin feeding on serum prolactin and gonadotropin and the onset of seasonal estrous cyclicity in sheep. Endocrinology 110, 1766-1772.
Kennaway, D.J., Dunstan, E.A., Gilmore, T.A. \& Seamark, R.F. (1984) Melatonin feeding increases ovulation rate in ewes. Proc. 7th Int. Congr. Endocrinology, Quebec City, Abstract no. 21.

Lincoln, G.A. \& Ebling, F.J.P. (1985) Effect of constantrelease implants of melatonin on seasonal cycles in reproduction, prolactin secretion and moulting in rams. J. Reprod. Fert. 73, 241-253.

Nett, T.M. \& Niswender, G.D. (1982) Influence of exogenous melatonin on seasonality of reproduction in sheep. Theriogenology 17, 645-651.

Nowak, R. \& Rodway, R.G. (1985) Effect of intravaginal implants of melatonin on the onset of ovarian activity in adult and prepubertal ewes. J. Reprod. Fert. 74, 287-293.

Poulton, A.L., English, J., Symons, A.M. \& Arendt, J. (1986) Effects of various melatonin treatments on plasma prolactin concentrations in the ewe. $J$. Endocr. 108, 287-292.

Sauer, M.J., Foulkes, J.A., Worsfold, A. \& Morris, B.A. (1986) Use of progesterone 11-glucuronide alkaline phosphatase conjugate in a sensitive microtitre-plate enzymeimmunoassay of progesterone in milk and its application to pregnancy testing in dairy cattle. $J$. Reprod. Fert. 76, 375-391.

Telfer, S.B. (1984) Controlled release glass (CRG)-its action and application in sheep. Proc. Sheep Veterinary Society 8, 82-85.

Received 16 September 1986 